\title{
Dieta cetogênica como terapêutica na epilepsia Refratária em crianças e adolescentes
}

\author{
Ketogenic diet as therapy in refractory epilepsy in children and adolescents \\ Dieta cetogénica como terapia en la epilepsia refractaria en niños y adolescentes
}

Recebido: 27/11/2021 | Revisado: 01/12/2021 | Aceito: 10/12/2021 | Publicado: 18/12/2021

Carolina Pereira Coelho
ORCID: https://orcid.org/0000-0002-5034-7497

Centro Geraldo Di Biase, Brasil

E-mail: carolinacoelhoo@gmail.com

Sávio Andrei Ricardo de Almeida Guedes

ORCID: https://orcid.org/0000-0001-9539-4791

Centro Geraldo Di Biase, Brasil

E-mail: Savioandrei123456789@gmail.com

Anastácia Mariana da Costa Melo

ORCID: https://orcid.org/0000-0001-9695-2919

Centro Universitário Geraldo Di Biase, Brasil

E-mail: anastaciamariana@yahoo.com.br

Cyntia Ferreira de Oliveira

ORCID: https://orcid.org/0000-0003-1724-0839

Centro Universitário Geraldo Di Biase, Brasil

E-mail: cyntia.ferreira.oliveira@gmail.com

Adriana Lau da Silva Martins

ORCID: https://orcid.org/0000-0002-0851-5522

Centro Universitário Geraldo Di Biase, Brasil

E-mail:adralmartins@hotmail.com

Aline Cristina Teixeira Mallet

ORCID: https://orcid.org/0000-0002-1789-0279

Centro Universitário Geraldo Di Biase, Brasil

E-mail: alinemallet@ugb.edu.br

Aline Miyoko Sakaguchi Yamashita

ORCID: https://orcid.org/0000-0002-8195-0416 Centro Geraldo Di Biase, Brasil

E-mail: alinemsy@gmail.com

\begin{abstract}
Resumo
A dieta cetogênica é caracterizada por ser uma dieta rica em gordura, pobre em carboidratos e moderada em proteínas. A epilepsia é definida como um distúrbio neurológico crônico tendo como característica convulsões recorrentes e não provocadas e a epilepsia refratária acontece quando os medicamentos não reduzem as crises de forma significativa. $\mathrm{O}$ objetivo desse trabalho é avaliar a dieta cetogênica clássica no tratamento da epilepsia refratária em crianças e adolescentes. A metodologia usada baseia-se em uma revisão de literatura, onde a coleta de dados foi feita utilizandose artigos, teses e sites. Os resultados encontrados indicam que a dieta cetogênica demonstra ser uma alternativa segura e eficaz. No entanto, existem efeitos colaterais e limitações relacionadas às resistências e dificuldades dos responsáveis que podem comprometer a eficácia do tratamento. Para o sucesso da dieta é importante que haja a cooperação dos familiares, o acompanhamento por uma equipe multidisciplinar durante todo o tratamento, e a atuação do nutricionista é de suma importância.
\end{abstract}

Palavras-chave: Epilepsia refratária; Dieta para epilepsia; Dieta cetogênica clássica; Epilepsia refratária infantil.

\begin{abstract}
The ketogenic diet is characterized by being a high-fat, low-carbohydrate, and moderate-protein diet. Epilepsy is defined as a chronic neurological disorder characterized by recurrent and unprovoked seizures and refractory epilepsy occurs when medications do not control the seizures. The aim of this work is to verify the effectiveness of the classic ketogenic diet in the treatment of refractory epilepsy in children and adolescents. The methodology used is based on a literature review, where data collection was carried out using articles, theses and websites. The results found indicate that the ketogenic diet proves to be a safe and effective alternative. However, there are side effects and limitations related to the resistance and difficulties of guardians that can compromise the effectiveness of the treatment. For the success of the diet, it is important to have the cooperation of family members, monitoring by a multidisciplinary team throughout the treatment, and the role of the nutritionist is of paramount importance.
\end{abstract}

Keywords: Refractory epilepsy; Diet for epilepsy; Classic ketogenic diet; Childhood refractory epilepsy. 


\section{Resumen}

La dieta cetogénica se caracteriza por ser una dieta alta en grasas, baja en carbohidratos y moderada en proteínas. La epilepsia se define como un trastorno neurológico crónico caracterizado por convulsiones recurrentes y no provocadas, y la epilepsia refractaria ocurre cuando los medicamentos no reducen significativamente las convulsiones. El objetivo de este trabajo es evaluar la dieta cetogénica clásica en el tratamiento de la epilepsia refractaria en niños y adolescentes. La metodología utilizada se basa en una revisión de la literatura, donde la recolección de datos se realizó mediante artículos, tesis y sitios web. Los resultados encontrados indican que la dieta cetogénica resulta ser una alternativa segura y eficaz. Sin embargo, existen efectos secundarios y limitaciones relacionados con las resistencias y dificultades de los tutores que pueden comprometer la eficacia del tratamiento. Para el éxito de la dieta, es importante contar con la colaboración de los familiares, el seguimiento de un equipo multidisciplinario durante todo el tratamiento, y el papel del nutricionista es de suma importancia.
\end{abstract}

Palabras clave: Epilepsia refractaria; Dieta para la epilepsia; Dieta cetogénica clásic; Epilepsia refractaria infantil.

\title{
1. Introdução
}

A epilepsia é um distúrbio neurológico crônico tendo como característica convulsões recorrentes e não provocadas, juntamente com atividade neuronal anormal e excessiva no cérebro, geralmente acompanhada de déficits cognitivos (Yang et. al., 2021). Acomete em torno de $1 \%$ da população mundial tendo maior incidência e prevalência em crianças e adolescentes (Costa et. al., 2020).

Em grande parte dos pacientes, o tratamento medicamentoso gera uma diminuição dos sinais e sintomas, proporcionando uma melhor qualidade de vida (Silva \& Cabral, 2008). O tratamento geralmente é iniciado com os Benzodiazepínicos, porém, se as convulsões persistirem, serão utilizadas drogas antiepilépticas (Kose et. al., 2018).

Mesmo com a utilização de medicamentos, cerca de 30\% dos pacientes ainda possuem convulsões não controladas, esta condição chama-se epilepsia refratária (Sampaio, 2016). Nesses casos, os pacientes se submetem a outros tratamentos, incluindo a estimulação do nervo vago, cirurgia após criteriosa avaliação e dietoterapia (Prezioso et. al., 2018).

Nestes casos, a dietoterapia tem como base a aplicação da dieta cetogénica (DC), que é utilizada desde 1921 para tratar a epilepsia, a DC, é uma das dietas mais estudadas como uma forma de tratamento para essa condição (Sampaio, 2018). É uma dieta caracterizada por ser rica em gordura, conter baixos níveis de carboidratos e possuir quantidade moderada de proteínas, ocasionando em um quadro de cetose (Su et. al., 2021).

A baixa ingestão de carboidratos faz com que o corpo humano passe por adaptações metabólicas aumentando a utilização de gorduras para a produção de energia, gerando os corpos cetônicos (CC) que parecem estar envolvidos no mecanismo terapêutico do controle das crises (Michalski et. al., 2019).

O presente trabalho teve como objetivo avaliar a dieta cetogênica clássica como tratamento para crianças e adolescentes portadoras de epilepsia refratária através de uma revisão de literatura de estudos publicados.

\section{Metodologia}

Trata-se de uma revisão narrativa de literatura (RNL), que possui caráter amplo e se propõe a descrever o desenvolvimento de determinado assunto, sob o ponto de vista teórico ou contextual, mediante a análise e interpretação da produção científica existente (Brum et al., 2015).

Por meio da busca avançada, utilizando-se os termos delimitadores de pesquisa, epilepsia refratária e dieta cetogênica para levantamento de dados dos últimos 17 anos. Este processo envolveu atividades de busca, identificação, fichamento de estudos, mapeamento e análise.

Os dados coletados para a seleção dos artigos analisados neste estudo atenderam aos seguintes critérios de inclusão: tratar-se de artigo original, ter resumo completo na base de dados no idioma de língua portuguesa ou inglesa, cujo objeto de estudo seja do interesse desta revisão narrativa e que esteja disponível gratuitamente, na íntegra, em formato eletrônico na base 
de dados, publicado nos últimos dezessete anos. Já os critérios de exclusão foram: artigo de revisão, tese ou dissertação e relato de experiências que fugissem fora do tema central.

Foram identificadas 15.372 publicações com o termo epilepsia refratária, 634 publicações com o termo epilepsia refratária infantil e 3.913 publicações com o termo dieta cetogênica, que foram incluídas de acordo com os critérios adotados neste estudo. Após a leitura dos resultados, verificou-se que somente 37 artigos eram de interesse do objeto de estudo proposto.

\section{Revisão Bibliográfica}

\subsection{Epilepsia}

A epilepsia é uma das doenças neurológicas mais comuns em pacientes mais jovens, sendo uma condição neurológica grave, caracterizada pela função anormal das células cerebrais, onde a mesma possui uma atividade excessiva, ocasionando na manifestação das crises epilépticas, o que compromete o controle dos músculos, comportamento e a consciência do indivíduo. (Porto et. al., 2007; Bailey et. al., 2021). As alterações na atividade cerebral podem ser detectadas pelo eletroencefalograma (EEG), esse exame permite determinar se as crises epilépticas são focais, ou seja, restrito a uma região cerebral ou generalizada, quando há alteração por todo encéfalo (Figura 1).

Figura 1. Representação do eletroencefalograma.

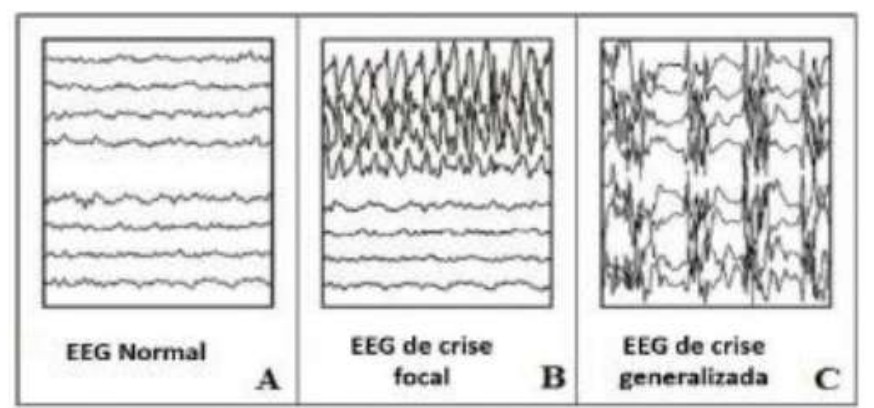

Resultado de um exame de eletroencefalograma (EEG) com resultado normal (A), de crise focal (B) e de crise generalizada (C). Fonte: (Lima; Negreiros, 2021)

A epilepsia refratária é caracterizada pela persistência na frequência das crises epilépticas após o uso de pelo menos duas medicações indicadas (Sampaio, 2018). Estima-se que 30\% dos pacientes com epilepsia não conseguem se livrar das crises com os medicamentos escolhidos, portanto, devem ser considerados resistentes aos medicamentos, sendo assim candidatos às terapias não farmacológicas, como cirurgia, estimulação do nervo vago e o tratamento com a dieta cetôgenica (DC) (Wrede et. al., 2021).

\subsection{Dieta cetogênica clássica na epilepsia refratária}

A DC é a dieta mais estudada como forma de tratamento para a epilepsia refratária, é uma dieta composta por alto teor de gordura, baixo teor de carboidratos e moderada quantidade de proteína, forçando o organismo a utilizar gordura como fonte primária de energia (Sampaio, 2018). A dieta cetogênica clássica (DCC) é a mais usada em casos de epilepsia refratária, a proporção dessa dieta é de 4:1 (significa que há 4 gramas de gordura para cada grama do somatório de proteína e carboidrato), desse modo, $90 \%$ das calorias são provenientes da gordura e 10\% da soma de proteína e carboidrato (Schoeler \& Cross, 2016).

Proporções mais baixas como 3:1 ou 2:1 (87\% e $81 \%$ de gordura na dieta respectivamente), podem ser usadas em pacientes em que uma proporção maior de proteínas e carboidratos é desejada, devido a melhor tolerabilidade ou para amenizar 
os efeitos colaterais (Kossof; Wang, 2013). As razões determinadas para a dieta devem ser seguidas rigorosamente, para isso, é necessário a pesagem dos alimentos que compõem o plano alimentar do paciente (Vaccarezza et. al., 2014).

O tratamento pode ser iniciado de duas maneiras: ambulatorial ou com internação. Na forma ambulatorial, a proporção dos macronutrientes são mudadas gradativamente em consultas, e o tratamento é feito em domicílio. E, com a internação, geralmente o paciente faz um jejum prévio e posteriormente inicia-se a dieta (Sampaio, 2018). Todavia, a eficácia no controle das crises epilépticas é a mesma, com ou sem a realização do jejum no início (Bergqvist et. al., 2005).

A dieta pode ser feita por dois a três anos sem interrupção, se necessário o nutricionista pode fazer alguns ajustes na dieta, o tempo do tratamento vai variar de acordo com a resposta de cada paciente, nesse período são feitos exames regularmente, como a dosagem de eletrólitos, perfil lipídico, hemograma, entre outros (Veronique et. al., 2015; RezaeI et. al., 2019). A eficácia clínica da DC como tratamento é significativa quando a redução das crises epilépticas é de, pelo menos, $50 \%$ (Kinderen et. al., 2011).

Cabe ressaltar, que, a aplicação da DC não dispensa o uso de medicamentos, as medicações antiepilépticas são mantidas, podendo haver a troca de algumas formulações, como a substituição de medicações que contém açúcar por comprimidos livres de carboidrato na composição (Sampaio, 2018). É importante destacar a importância da suplementação de micronutrientes para o equilíbrio nutricional das crianças e adolescentes que estão em tratamento, decorrente da baixa ingestão de verduras, frutas e alimentos que contêm vitaminais e minerais.

\subsection{Corpos cetônicos}

Com a diminuição da ingestão de carboidratos e proteínas, o fígado é estimulado a produzir e liberar corpos cetônicos (CC) na circulação para gerar energia, sendo este processo chamado de cetose (Ma et. al.; 2019). Os ácidos graxos ingeridos através da DC sofrem metabolização nas mitocôndrias do fígado, gerando os corpos cetônicos denominados acetoacetato, acetona e $\beta$-hidroxibutirato, posteriormente, os corpos cetônicos são exportados do fígado através da corrente sanguínea para diferentes tecidos, inclusive para o cérebro atravessando a barreira hematoencefálica (Mcnally \& Hartman, 2012). Sugere-se que dentre de três a quatro semanas o cérebro já esteja adaptado e os neurônios passam a utilizar os corpos cetônicos como principal fonte energética ao invés da glicose (Prezioso et. al., 2018).

Apesar dos mecanismos pelos quais a DC ajuda no controle das crises não serem completamente compreendidos, a eficácia da ação terapêutica da dieta tem sido associada com a produção dos corpos cetônicos (Lima et. al., 2014).

A concentração de neurotransmissores é o mecanismo mais estudado, nesse caso, os CC tem a capacidade de modular os neurotransmissores, influenciando nas suas concentrações, os principais neurotransmissores envolvidos é o glutamato e o ácido $\gamma$-aminobutírico (neurotransmissor excitatório e inibitório respectivamente) (Chen \& Kossoff, 2012). Nessa situação, há o aumento do ácido $\gamma$-aminobutírico e redução do glutamato.

Outro mecanismo sugerido, é que os CC podem modular os transportadores vesiculares de glutamato (VGLUT), que são responsáveis pelo preenchimento de vesículas pré-sinápticas com esse neurotransmissor. Diante disso, há uma alteração no armazenamento de glutamato nas vesículas, influenciando a sua posterior liberação, levando a uma diminuição desse neurotransmissor excitatório pela inibição do VGLUT (Juge et. al., 2010).

Outra hipótese, é que os CC modulam algumas aminas biogênicas, com maior destaque para a adenosina que é um neuromodulador inibitório presente no sistema nervoso central, assim, há o aumento na concentração de adenosina e ativação do seu receptor, reduzindo a excitabilidade (Yudkoff et. al., 2005; Yoon et. al., 2014).

$\mathrm{O}$ aumento da inibição ou redução da excitabilidade, pode influenciar a função do cérebro, interferindo nas crises epilépticas. A diminuição de glicose obtida em uma dieta cetogênica, ativa canais que são sensíveis ao ATP (KATP) levando a hiperpolarização neuronal, contribuindo assim, para o controle da excitação neuronal (Maalouf et. al., 2009). De forma geral, 
todos esses mecanismos citados estão envolvidos na redução da excitabilidade, o que é algo positivo visto que na epilepsia as células cerebrais possuem uma atividade excessiva.

Os possíveis mecanismos antiepilépticos proporcionado pelo tratamento com a dieta cetogênica foram resumidos na Figura 2.

Figura 2. Possíveis mecanismos de ação da dieta cetogênica.

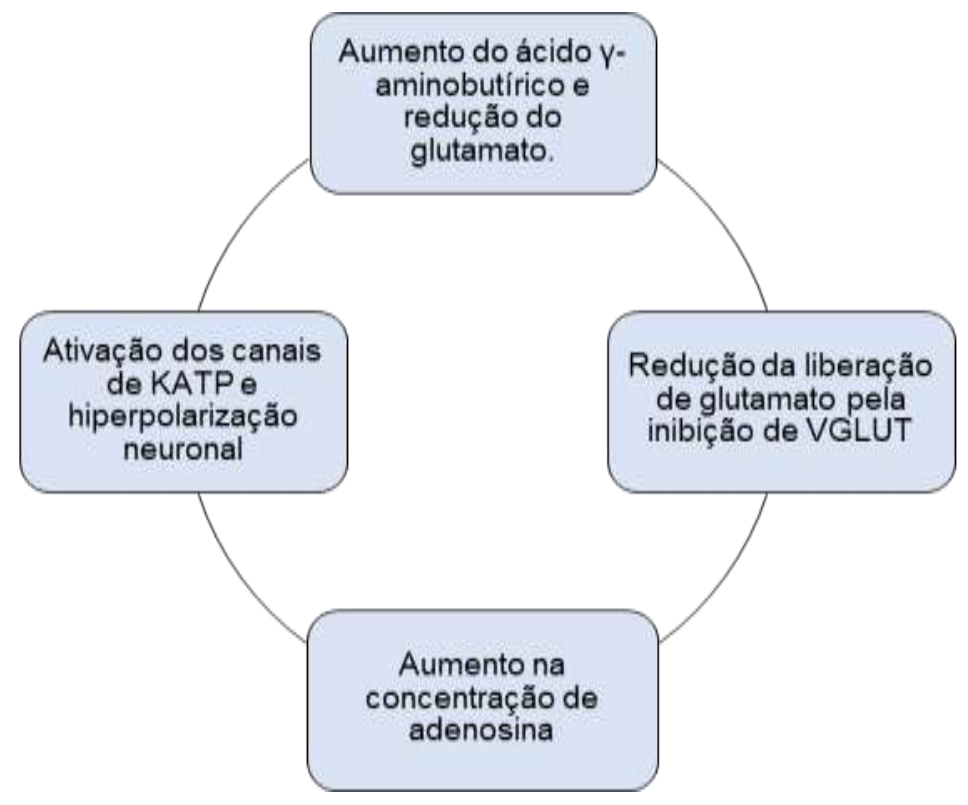

Fonte: Adaptado de Neves (2019).

\section{Resultados e Discussões}

Foram selecionados 8 trabalhos, 6 de autores nacionais e 2 de autores internacionais, com formas de estudos e tamanhos amostrais diversos. Neste trabalho foram incluídos estudo clínico, longitudinal, retrospectivo e estudo clínico randomizado (Quadro 1). 
Quadro 1. Análise dos estudos que avaliaram a eficácia da dieta cetogênica.

\begin{tabular}{|c|c|c|c|c|}
\hline Autores & Tipo de estudo & Pacientes alvo & Metodologia & Resultados \\
\hline $\begin{array}{l}\text { Freitas et. al., 2007, } \\
\text { Brasil }\end{array}$ & Estudo Longitudinal & $\begin{array}{l}54 \text { crianças com } \\
\text { epilepsia refratária, } \\
\text { com idade entre } 1 \text { a } \\
12 \text { anos. }\end{array}$ & $\begin{array}{l}\text { Aplicação da dieta } \\
\text { cetogênica clássica. }\end{array}$ & $\begin{array}{l}\text { Em } 2 \text { meses de tratamento ( } 54 \text { pacientes) } \\
\text { - } 30 \text { tiveram > 75\% de redução das crises. } \\
\text { - } 17 \text { tiveram entre } 50-75 \% \text { de redução. } \\
\text { - } 7 \text { tiveram < 50\% de redução. } \\
\text { Em } 2 \text { anos de tratamento ( } 29 \text { pacientes) } \\
\text { - } 18 \text { tiveram > 75\% de redução das crises. } \\
\text { - } 11 \text { tiveram entre } 50-75 \% \text { de redução. } \\
\end{array}$ \\
\hline $\begin{array}{l}\text { Ramos et. al., 2004, } \\
\text { Brasil }\end{array}$ & Estudo Longitudinal & $\begin{array}{l}12 \text { Pacientes de } 4 \text { a } \\
17 \quad \text { anos, com } \\
\text { epilepsia refratária. }\end{array}$ & $\begin{array}{lll}\text { Aplicação da } & \text { dieta } \\
\text { cetogênica clássica. } & \end{array}$ & $\begin{array}{l}\text { Em } 3 \text { meses de tratamento (12 pacientes) } \\
\text { - } 5 \text { tiveram uma redução > 70\% das crises. } \\
\text { - } 3 \text { tiveram >50\% de redução. } \\
\text { - } 4 \text { tiveram uma redução <50\%. } \\
\text { Em } 6 \text { meses de tratamento (12 pacientes) } \\
\text { - } 6 \text { tiveram uma redução >70\%. } \\
\text { - } 4 \text { tiveram >50\% de redução. } \\
\text { - } 2 \text { tiveram uma redução <50\%. } \\
\text { Em } 1 \text { ano de tratamento (8 pacientes) } \\
\text { - } 6 \text { tiveram }>70 \% \text { de redução. } \\
\text { - } 2 \text { tiveram } 90 \% \text { de redução. }\end{array}$ \\
\hline $\begin{array}{l}\text { Sampaio et. al., 2017, } \\
\text { Brasil }\end{array}$ & $\begin{array}{l}\text { Estudo } \\
\text { Clínico }\end{array}$ & $\begin{array}{l}10 \text { pacientes com } \\
\text { epilepsia refratária, } \\
\text { de } 9 \text { meses a } 16 \text { anos. }\end{array}$ & $\begin{array}{l}\text { Aplicação da dieta } \\
\text { cetogênica clássica. }\end{array}$ & $\begin{array}{l}\text { Em } 3 \text { meses de tratamento } \\
\text { - } 6 \text { tiveram }<50 \% \text { de redução das crises. } \\
\text { - } 3 \text { tiveram }>50 \% \text { de redução. } \\
\text { - } 1 \text { teve } 100 \% \text { de redução. } \\
\end{array}$ \\
\hline $\begin{array}{l}\text { Barros et. al., 2006, } \\
\text { Brasil }\end{array}$ & $\begin{array}{l}\text { Estudo } \\
\text { Clinico }\end{array}$ & $\begin{array}{l}10 \text { pacientes com } \\
\text { epilepsia refratária, } \\
\text { com idade entre } 2 \text { e } \\
18 \text { anos. }\end{array}$ & $\begin{array}{l}\text { Aplicação da DC clássica. } \\
\text { O tempo proposto da dieta } \\
\text { era de } 6 \text { meses. }\end{array}$ & $\begin{array}{l}\text { - } 1 \text { teve }>90 \% \text { da redução das crises. } \\
\text { - } 2 \text { tiveram } 100 \% \text { de redução. } \\
\text { - } 2 \text { tiveram entre } 50 \% \text { e } 90 \% \text { de redução } \\
\text { - } 2 \text { tiveram menos que } 50 \% \text { de redução. } \\
\text { - } 3 \text { tiveram aumento das crises. } \\
\end{array}$ \\
\hline $\begin{array}{l}\text { Vasconcelos et. al., } \\
\text { 2004, Brasil }\end{array}$ & $\begin{array}{l}\text { Estudo } \\
\text { Retrospectivo }\end{array}$ & $\begin{array}{l}6 \text { pacientes entre } 1 \mathrm{e} \\
15 \text { anos, que fizeram } \\
\text { o tratamento com a } \\
\text { DC clássica. }\end{array}$ & $\begin{array}{l}\text { Análise aos prontuários } \\
\text { médico dos pacientes. A } \\
\text { duração do tratamento } \\
\text { durou até } 4 \text { anos. }\end{array}$ & $\begin{array}{l}\text { - } 3 \text { tiveram redução }>50 \% \text { nas crises. } \\
\text { - } 2 \text { pacientes tiveram } 100 \% \text { de redução } \\
\text { - } 1 \text { paciente teve piora das crises (ele saiu da dieta em } 2 \\
\text { momentos). }\end{array}$ \\
\hline $\begin{array}{l}\text { RizzuttI et. al., 2006, } \\
\text { Brasil }\end{array}$ & $\begin{array}{l}\text { Estudo } \\
\text { Longitudinal }\end{array}$ & $\begin{array}{l}23 \text { pacientes com } \\
\text { idade entre } 2 \text { e } 17 \\
\text { anos, com epilepsia } \\
\text { refratária. }\end{array}$ & $\begin{array}{l}\text { Aplicação da DC clássica } \\
\text { com duração de } 1 \text { ano }\end{array}$ & $\begin{array}{l}\text { - } 3 \text { tiveram } 100 \% \text { de redução. } \\
\text { - } 5 \text { tiveram >90\% de redução. } \\
\text { - } 6 \text { tiverem entre } 50 \% \text { e } 90 \% \text {. } \\
\text { - } 7 \text { tiverem < } 50 \% \text { de redução. } \\
\text { - } 2 \text { não tiverem efeitos; }\end{array}$ \\
\hline Seo et. al., 2007, Coreia & $\begin{array}{l}\text { Estudo } \\
\text { Clínico randomizado }\end{array}$ & $\begin{array}{l}76 \text { pacientes com } \\
\text { epilepsia refratária, a } \\
\text { média de idade foi de } \\
3 / 4 \text { anos. }\end{array}$ & $\begin{array}{l}40 \text { pacientes utilizaram a } \\
\text { DC } 4: 1 \text { e } 36 \text { pacientes a } \\
\text { DC } 3: 1\end{array}$ & $\begin{array}{l}\text { - } 22 \text { dos pacientes com a DC 4:1 tiveram } 100 \% \text { de redução. } \\
\text { - } 11 \text { dos pacientes com a DC 3:1 tiveram } 100 \% \text { de redução. } \\
12 \text { pacientes que não se livraram das crises com } 3: 1 \text {, mudaram } \\
\text { para 4:1 e } 4 \text { deles teve mais de } 90 \% \text { de redução das crises, No } \\
\text { geral, } 75 \% \text { dos pacientes mostraram redução adicional das crises } \\
\text { de mais de } 50 \% \text { após alterar a proporção cetogênica. }\end{array}$ \\
\hline $\begin{array}{l}\text { Kang et. al., 2005, } \\
\text { Coreia }\end{array}$ & Estudo Longitudinal & $\begin{array}{l}175 \text { pacientes com } \\
\text { epilepsia refratária } \\
\text { com } 6 \text { meses a } 17 \\
\text { anos }\end{array}$ & $\begin{array}{l}\text { Aplicação da dieta } \\
\text { cetogênica clássica. }\end{array}$ & $\begin{array}{l}\text { Em } 3 \text { meses de tratamento (175 pacientes) } \\
\text { - } 53 \text { tiveram uma redução > 50\% das crises. } \\
\text { - } 70 \text { tiveram } 100 \% \text { de redução. } \\
\text { - } 52 \text { tiveram uma redução <50\% } \\
\text { Em } 6 \text { meses de tratamento (136 pacientes) } \\
\text { - } 45 \text { tiveram uma redução > } 50 \% \\
\text { - } 66 \text { tiveram } 100 \% \text { de redução. } \\
\text { - } 25 \text { tiveram uma redução < } 50 \% \text {. } \\
\text { Em } 1 \text { ano de tratamento (91 pacientes) } \\
\text { - } 32 \text { tiveram uma redução > } 50 \% \text {. } \\
\text { - } 50 \text { tiveram } 100 \% \text { de redução. } \\
\text { - } 9 \text { tiveram uma redução < } 50 \% \text {. }\end{array}$ \\
\hline
\end{tabular}

Fonte: Autores (2021).

O Quadro 1, apresenta os resultados obtidos pelos pacientes que foram submetidos ao tratamento com a dieta cetogênica clássica.

$\mathrm{Na}$ análise feita, pode-se observar que existem benefícios da aplicação da DCC como um meio de tratamento para a epilepsia refratária em crianças e adolescentes, sendo assim, um tratamento que pode ser eficaz. Porém, a resposta ao tratamento é individual, uns podem ter resultados melhores que outros, devido a diversos fatores, tais como: características individuais dos pacientes, alimentar-se fora do plano alimentar prescrito, ocorrências de efeitos colaterais que comprometem a aderência do paciente, e isso foi observado nos trabalhos analisados. 
No estudo feito por Freitas et. al., 2007, a DCC provou ser um tratamento eficaz. Alguns pacientes abandonaram o tratamento por intolerância a dieta, devido aos efeitos colaterais, como: náuseas, vômitos e hipoglicemia. Porém, todos apresentaram melhoras na cognição.

No trabalho de Ramos et. al., 2004 também foi observado benefícios da aplicação da DCC pelos autores. A melhora na cognição e nas atividades motoras foi observada em todos os pacientes.

No estudo feito por Sampaio et. al., 2017, os pacientes tiveram constipação e sonolência como efeito colateral, mas, 8 deles tiveram melhorias na qualidade de vida. Os autores finalizaram, dizendo que, a dieta cetogênica melhora a qualidade de vida em crianças e adolescentes com epilepsia refrataria, sendo assim, uma terapêutica eficaz.

Ao longo do tratamento no estudo de Barros et. al., 2006, também houveram desistências por limites impostos pela dieta ou por preocupação do pais frente aos efeitos colaterais apresentados pelos os seus filhos, como: constipação, desidratação e recorrências das crises epilépticas.

Na pesquisa de Vasconcelos et. al., 2004, também observou-se benefícios do tratamento com a DCC. Um paciente teve piora das crises, é importante destacar que esse paciente saiu da dieta em dois momentos diferentes, o que pode ter influenciado em seu resultado. Os autores finalizaram dizendo que sempre que for viável a DCC deve ser aplicada como tratamento nas crianças portadoras da epilepsia refratária.

No estudo de Rizzutti et. al., 2006, os efeitos colaterais observados foram constipação, náuseas, vômitos, sonolência e hiperlipidemia. Nessa pesquisa, foi concluído que a DCC pode ser considerada eficaz para o tratamento de epilepsia refratária em crianças e adolescentes, também foi destacado que é importante a cooperação dos pais.

Para a realização do trabalho de Seo et al., 2007, as crianças foram divididas em dois grupos, onde 40 pacientes ficaram com a proporção 4:1 (90\% de gordura) e 36 pacientes com a dieta 3:1 (87\% de gordura), para que fosse possível comparar a dieta 3:1 com a 4:1 e analisar os benefícios, efeitos colaterais e tolerabilidade. Dos 40 pacientes na dieta 4:1, 22 deles obtiveram redução de 100\% no número de crises e dos 36 com DC 3:1, 11 obteve o mesmo resultado.

Observou-se uma melhor tolerabilidade na dieta 3:1, devido aos efeitos gastrointestinais que ocorreram mais na proporção 4:1. É importante dizer que, 12 pacientes que não se livraram das crises com 3:1, alternaram a proporção para 4:1 e 4 deles tiveram mais que $90 \%$ de diminuição das crises. No geral, 9 desses 12 pacientes tiveram redução adicional das crises de mais de 50\% após essa mudança. Os autores finalizaram dizendo que os resultados na proporção 4:1 foram melhores na redução das crises quando comparados ao grupo das crianças tratadas com a dieta 3:1.

Na análise de Kang et. al.,2005, as causas do abandono do tratamento foram desde falecimentos até intolerância em seguir a dieta, por mais que, a causa do falecimento não tenha sido diretamente pela dieta cetogênica, é importante haver uma atenção criteriosa sobre as possíveis consequências do tratamento.

Apesar de alguns pacientes não terem a redução no número de crises, eles tiveram uma melhora na cognição, melhorando a qualidade de vida. Diante disso, os benefícios da DC, vão além de reduzir a frequência das crises (Berkel et. al., 2018).

\subsection{Desistência do tratamento}

A resistência ao tratamento dos pacientes e familiares e a desistência são os grandes motivos da ineficácia da dieta cetogênica como terapêutica (Pereira et. al., 2009). Antes de iniciar, é importante alertar aos familiares que, a resposta a dieta é individual, pode haver diminuição dos episódios das crises, remissão total ou até mesmo nem haver resposta ao tratamento (Santos et. al., 2019).

A partir das análises dos estudos, foi possível perceber que a desistência tinha como um dos principais motivos os efeitos colaterais que ocorriam ao longo do tratamento. 


\subsection{Efeitos colaterais}

A constipação é um efeito bem comum, sendo o resultado da diminuição do volume ingerido de alimentos que contém fibras, mas, com a reintrodução das fibras e uma ingestão hídrica adequada, há uma melhora nesse quadro (GuzeL et al., 2018). Por outro lado, os pacientes também podem apresentar diarreia, o que pode ser devido ao aumento de gorduras na dieta que supera a capacidade digestiva. O mesmo ocorre para as náuseas e vômitos, que decorre em parte, ao excesso de gorduras, que lentifica o esvaziamento gástrico podendo levar a esses sintomas (Giner, 2017).

A sonolência e os sinais de baixa energia também é recorrente, sendo o resultado da hipoglicemia e da ausência de transportadores de corpos cetônicos (Giner, 2017). A hipoglicemia é caracterizada pela redução das taxas de açúcar no sangue, que pode responder facilmente a ingestão de alimentos que tenham glicose, o que leva o reestabelecimento da glicemia. $\mathrm{O}$ tempo que o organismo leva para se acostumar a utilizar gordura e os CC como fonte primária de energia é chamado de cetoadaptação, e durante essa adaptação não há a quantidade ideal de transportadores de CC, causando a falta de energia e a sonolência nos pacientes. (Maia, 2020).

A hiperlipidemia pode acontecer em qualquer momento durante o tratamento com a DC, para isso, é importante que o Nutricionista faça ajustes nas refeições, dando preferência a gorduras menos saturadas, visto que o excesso de gordura saturada está relacionada a um perfil lipídico inadequado, se não houver melhora, deve-se considerar reduzir a proporção da DC e ir acompanhando as taxas lipídicas através de exames laboratoriais (Bergqvist, 2012).

A desidratação pode ocorrer pois os corpos cetônicos são ácidos que ultrapassam a capacidade de tamponamento do sangue, e o organismo tenta compensar pela maior excreção urinária de eletrólitos e água, que se for associada à menor ingestão hídrica pode resultar na desidratação (Barros et. al., 2006). É, importante assegurar que o paciente receba a quantidade de líquido recomendada, a quantidade de líquidos deve ser calculada de acordo com as necessidades basais (Lin et. al, 2017).

A falta de eficácia do tratamento ocasionando nas crises epilépticas recorrentes pode ser justificada pela utilização de medicamentos contendo açúcar, alimentar-se fora da dieta proposta, alterações indevidas no plano alimentar. Para solucionar isso, pode-se utilizar de algumas estratégias, como aumentar a proporção cetogênica, fazer um jejum de 12 horas, mas é essencial que haja uma análise criteriosa sobre o como está a alimentação e saúde do paciente antes de qualquer modificação (Kossoff et. al., 2016).

\section{Considerações Finais}

De acordo com os diversos estudos expostos e discutidos, percebeu-se que a dieta cetogênica constitui-se uma alternativa eficaz para o tratamento de pacientes com epilepsia refratária. Porém, precisa haver a consciência dos potenciais efeitos colaterais e detectá-los precocemente para que haja uma correção, evitando danos maiores, e garantindo a adesão ao tratamento. E, sempre antes de iniciar o tratamento, a equipe responsável deve estudar o paciente por completo, para que se possa saber como está a saúde do paciente.

Cabe ressaltar, que, não só os pacientes, mas todos os seus familiares devem participar ativamente do tratamento, isso é essencial para que o mesmo se sinta acolhido. Além do mais, os responsáveis pelas crianças e adolescentes precisam ter conhecimento sobre a DC, para garantir que o tratamento seja feito da forma correta e conhecer os efeitos que podem ocorrer com a aplicação da dieta, para que eles se preparem para lidar com essas situações.

Precisa-se destacar o quanto é importante que haja o acompanhamento de uma equipe composta por vários profissionais de diferentes áreas, com destaque ao nutricionista, que irá avaliar o paciente em todas as fases do tratamento, através de uma avaliação nutricional completa, prescrição da dieta e suplementos, orientação adequada em relação a alimentação aos responsáveis dos pacientes, tendo assim, uma melhor qualidade de vida, tanto durante, quanto após a suspensão da dieta. 
Por fim, estudos futuros devem ser idealizados para que novas pesquisas esclareçam o real mecanismo de ação da dieta cetogênica possibilitando a obtenção de novas formas de sua aplicação.

\section{Referências}

Bailey, J., West, M., Agarwal, R. \& Kumar, G. (2021). Measuring the Barriers to Adherence with Neurology Clinic Appointments for Children With Epilepsy: a Pilot Study. Child Neurology Open. 8, 1-8.

Barros, C. R. M. R. (2006). Dieta cetogênica utilizando jejum fracionado: emprego ambulatorial em epilepsia refratária. Dissertação (mestrado) - Curso de Medicina e Ciências da Saúde, com Área de Concentração em Neurociências, Pontifícia Universidade Católica do Rio Grande do Sul, Porto Alegre.

Bergqvist, A. G. C. (2012). Long-term monitoring of the ketogenic diet: Do’s and Don'ts. Epilepsy Res. 100(3), 261-6.

Bergqvist, A. G., Schall, J. I., Gallagher, P. R.;,Cnaan, A. \& Stallings, V. A. (2005). Fasting versus gradual initiation of the ketogenic diet: a prospective, randomized clinical trial of efficacy. Epilepsia. 46(11), 1810-9.

Berkel, A. A. V., Ijff, D. M. \& Verkuyl, J. M. (2018). Cognitive benefits of the ketogenic diet in patients with epilepsy: a systematic overview. Epilepsy \& Behavior. 87(0), 69-77.

Brum, C. N. de., Zuge, S. S., Rangel, R. F., Freitas, H. M. B de., Pieszak, G. M. (2015). Revisão narrativa da literatura: aspectos conceituais e metodológicos na construção do conhecimento da enfermagem. In: Lacerda, M. R., Costenaro, R. G. S. Metodologia de pesquisa para a enfermagem a saúde da teoria a prática. $1^{\mathrm{a}}$ ed. Porto Alegre: Moriá, 123-142.

Chen, W. \& Kossoff, E. H. (2012). Long-term follow-up of children treated with the modified Atkins diet. J Child Neurol. 27(6), 754-8.

Costa, L. L. O., Brandão, E. C. \& Segundo, L. M. B. M. (2020). Atualização em epilepsia: revisão de literatura. Rev Med, São Paulo. 99(2), $170-81$.

Freitas, A., Paz, J. A., Casella, E. B. \& Marques-Dias, M. J. (2007). Ketogenic diet for the treatment of refractory epilepsy: a 10 year experience in children, Arq Neuro Psiquiatr, 65(2b), 381-384.

Giner, C. P. (2017). Manual para o uso da dieta cetogênica. Madrid: Nutricia.

Guzel, O., Uysal, U. \& Arslan, N. (2018). Efficacy and tolerability of olive oil-based ketogenic diet in children with drug-resistant epilepsy: a single center experience from turkey. Eur j paediatr neurol. 23(1), 143-151.

Juge, N., Gray, J. A., Omote, H., Miyaji, T., Inoue, T., Hara, C., Uneyama, H., Edwards, R. H., Nicoll, R. A. \& Moriyama, Y. (2010). Metabolic control of vesicular glutamate transport and release. Neuron. 68(1), 99-112.

Kang, H. C., Kim, Y. J., Kim, D. W. \& Kim, H. D. (2005). Efficacy and safety of the ketogenic diet for intractable childhood epilepsy: Korean multicentric experience. Epilepsia. 46(2), 272-279.

Kinderen, R. J. A., Lambrechts, D. A. J. E., Postulart, D., Kessels, A. G. H., Hendriksen, J. G. M., Albert, P., Aldenkamp, A. P., Evers, S. M. A. A. \& Majoie, M. H. J. M. (2011). Research in to the (Cost-) effectiveness of the ketogenic diet among children and adolescents with intractable epilepsy: design of a randomized controlled trial. BMC Neurol. 11(10), 1-10.

Kose, E., Guzel, O. \& Arslan, N. (2018). Analysis of hematological parameters in patients treated with ketogenic diet duetodrug-resistant epilepsy. Neurol Sci. $39(1), 85-89$

Kossoff, E. H. \& Wang, H. S. (2013). Dietary Therapies for Epilepsy. Biomed J. 36(1), 2-8.

Kossoff, E. H., Turner, Z., Doerrer, S., Cervenka, M. C. \& Henry, B. J. (2016). The ketogenic and modified atkins diet. Demos.

Lima, G. S. S. \& Negreiros, G. S. (2021). Uso clínico do canabidiol em pacientes pediátricos com epilepsia. TCC (Graduação) - Curso de Farmácia, Universidade São Judas, São Paulo.

Lin, A., Turner, Z., Doerrer, S. C., Stanfield, A. \& Kossof, E. H. (2017). Complications During Ketogenic Diet Initiation: Prevalence, Treatment, and Influence on Seizure Outcomes. Pediatr Neurol. 68(0), 35-9.

Ma, S. \& Suzuki, K. (2019). Keto-Adaptation and Endurance Exercise Capacity, Fatigue Recovery, and Exercise-Induced Muscle and Organ Damage Prevention: A Narrative Review. Sports. 7(40), 1-10.

Maia, B. (2020). O que é ceto-adaptação? Disponível em: https://bernardomaia.com.br/o-que-e-ceto-adaptacao/. Acesso em: 20 jul. 2021.

Maalouf, M., Rho, J. M. \& Mattson, M. P. (2009). The neuro protective properties of calorie restriction, the ketogenic diet, and ketone bodies. Brain Research Reviews. 59(2), 293-315.

Mcnally, M. A. \& Hartman, A. L. (2012). Ketone bodies in epilepsy. Journal of Neurochemistry. 121(1), 28-35.

Michalski, K. D., Nowaczyk, P. M. \& Siedzik, K. (2019). Effect of a four-week ketogenic diet on exercise metabolism in CrossFit-trained athletes. Journal of the International Society of Sports Nutrition. 16(16), 1-15.

Neves, G. S. (2019). Efeito da dieta cetogênica (Atkins Modificada) sobre o perfil lipídico, parâmetros glicêmicos e controle de crises de pacientes adultos com epilepsias farmacorresistentes: um estudo exploratório. Dissertação (Mestrado) - Curso de Nutrição, Universidade Federal de Santa Catarina, Florianópolis. 
Pereira, E. S., Alves, M., Sacramento, T. \& Rocha, V. L. (2009). Dieta Cetogênica: Como o uso de uma dieta pode interferir em mecanismos neuropatológicos. Revista de Ciências Médicas e Biológicas.1(9), 78-82.

Porto, L. A., Siqueira, J. S., Seixas, L. N., Almeida, J. R. G. S. \& Quintans-Júnior, L. J. (2007). O papel dos canais iônicos nas epilepsias e considerações sobre as drogas antiepilépticas - uma breve revisão. J Epilepsy Clin Neurophysiol. 13(4), 69-175.

Prezioso, G., Carlone, G., Zaccara, G \&; Verrotti, A. (2018). Efficacy of ketogenic diet for infantile spasms: A systematic review. Acta Neurol Scand. 137(1), 4-11.

Ramos, A. M. F. (2004). Impacto nutricional da dieta cetogênica na epilepsia refratária infantil de difícil controle. Tese (Mestrado) - Curso em Ciências, Universidade Federal de São Paulo, S.P.

Rezaei, S., Abdurahman, A. A., Saghazadeh, A., Badv, R. S. \& Mahmoudi, M. (2019). Short-term and long-term efficacy of classical ketogenic diet and modified Atkins diet in children and adolescents with epilepsy: A systematic review and meta-analysis. Nutritional Neuroscience. 22(5), 317-334.

Rizzutti, S., Ramos, A. M. F., Cintra, I. P., Muszkat, M. \& Gabbai, A. A. (2006). Avaliação do perfil metabólico, nutricional e efeitos adversos de crianças com epilepsia refratária em uso da dieta cetogênica. Revista de Nutrição. 19(5), 573-579.

Sampaio, L. P. B. (2016). Dieta cetogênica para o tratamento da epilepsia. Arquivos de neuro-psiquiatria. São Paulo. 74(10), 842-848.

Sampaio, L. P. B., Takakura, C. \& Manreza, M. L. G. (2017). The use of a formula-based ketogenic diet in children with refractory epileps. Arq. Neuropsiquiatr. 75(4), 234-237.

Sampaio, L. P. B. (2018). ABC da dieta cetogênica para epilepsia refratária. Rio de Janeiro: DOC Content.

Santos, D. K., Errante, P. R. E., Pontes Junior, L. C. B., Raphael Junior, A., Souza, P. C., Ferraz, R. R. N. \& Rodrigues, F. S. M. (2019). Utilização da dieta cetogênica como estratégia para o manejo de pacientes com epilepsia refratária: uma revisão da literatura. International Journal of Health Management, 5(1), $1-8$.

Schoeler, N. E. \& Cross, J. H. (2016). Ketogenic dietary therapies in adults with epilepsy: a practical guide. Pract Neurol. 16(3), 208-214.

Seo, J. H., Lee, Y. M., Lee, J. S., Kang, H. C., Kim, H. D. (2007). Efficacy and Tolerability of the Ketogenic Diet According to Lipid: Nonlipid Ratios Comparison of 3:1 with 4:1 Diet. Epilepsia. 48(4), 801-805.

Silva, A. V. \& Cabral, F. R. (2008). Ictogênese, Epileptogênese e Mecanismo de Ação das Drogas na Profilaxia e Tratamento da Epilepsia. Journal of Epilepsy and Clinical Neuro Physiology. 14(2), 39-45.

Su, T. Y., Hung, P. L., Chen, C., Lin, Y. J. \& Peng, S. J. (2021). Theory-Based Electroencephalographic Connectivity and Its Association with Ketogenic Diet Effectiveness in Epileptic Children. Nutrients. 13(7), 1-15.

Vaccarezza, M. M., Toma, M. V., Guevara, J. D. R., Diez, C. G. \& Agosta, G. E. (2014). Treatment refractory epilepsy with the modified Atkins diet. ArchArgentPediatr. 112(4), 348-512.

Vasconcelos, M., Azevedo, P., Esteves, L., Brito, A., Olivaes, M. \& Herdy, G. (2004). Dieta cetogênica para epilepsia intratável em crianças e adolescentes: relato de seis casos. Rev. Assoc. Med. Bras. 50(4), 380-5.

Veronique, G., Schall, J. I., Stallings, V. A. \& Bergqvist, C. A. (2015). Long-term impact of the ketogenic diet on growth and resting energy expenditure in children with intractable epilepsy. National Institutes of Health. 56(9), 1-15.

Wrede, R.V., Helmstaedter, C. \& Surges, R. (2021). Cannabidiol in the Treatment of Epilepsy. Clinical Drug Investigation. 41(3), 211-220.

Yang, H., Chi, Y., Zhu, Z., Huang, K,Xiang, L., Xiao, B. \& Feng, L. (2021). Attitudes Toward Epilepsy Among Parents of Children with Epilepsy in Southern China. Front. Neurol. 11(0), 1-10.

Yoon, J. R., Lee, E. J., Kim, H. D. K., Lee, J. H. \& Kang, H. C. (2014). Polyunsaturated fatty acid-enriched diet therapy for a child with epilepsy. Brain Dev. $36(2), 163-166$

Yudkoff, M., Daikhin, Y., Nissim, I., Horyn, O., Lazarow, A., Luhovyy, B., Wehrli, S. \&_Nissim, I. (2005). Response of brain amino acid metabolism to ketosis. Neurochem Int. 47(1-2), 119-28. 